Homology, Homotopy and Applications, vol.19(1), 2017, pp.171-179

\title{
DUALITY IN THE HOMOLOGY OF 5-MANIFOLDS
}

\author{
W.H. MANNAN \\ (communicated by Graham Ellis)
}

\begin{abstract}
We show that the homological properties of a 5-manifold $M$ with fundamental group $G$ are encapsulated in a $G$-invariant stable form on the dual of the third syzygy of $\mathbb{Z}$. In this notation one may express an even stronger version of Poincaré duality for $M$. However, we find an obstruction to this duality.
\end{abstract}

\section{Introduction}

The purpose of this article is to consider a 5-manifold $M$ with finite fundamental group $G$, and examine the interaction between the module theory over $\mathbb{Z}[G]$ with the homology theory of the simply connected 5 -manifold $\tilde{M}$. Much of what we do holds more generally for $2 n+1$-manifolds with $n-1$-connected universal cover. However, we focus on 5-manifolds for clarity and in order to avoid such additional assumptions, as well as to highlight that low-dimensional phenomena to not impinge on our results.

We associate to $M$ an algebraic complex $C(M)$; the cellular chain complex for $\tilde{M}$ which one may regard as an algebraic complex over $\mathbb{Z}[G]$. This algebraic object (defined up to chain homotopy equivalence) is universal in the sense that all twisted homology/cohomology of $M$ may be extracted from it (by taking coefficients in the appropriate module over $\mathbb{Z}[G])$.

Using purely algebraic methods from our earlier paper [5], in $\S 2$ we show that we may take $C(M)$ to satisfy a certain duality, so that its modules and those of its dual $C(M)^{*}$ may be identified with four of the five differentials the same in both cases. We call this dual form. Note this does not imply $C(M) \cong C(M)^{*}$.

A self-dual (up to signs on boundary maps) polyhedral cell description for $M$ itself could be obtained from Lefschetz' classical proof of Poincaré Duality cf. [6, Theorem 2.1] or via geometric arguments cf [4, Theorem 6.5]. Our result differs in that we are working with the universal cover of $M$ and modules over $\mathbb{Z}[G]$. As mentioned earlier this object more completely represents the (co)homology of $M$.

In $\S 3$ we observe that when $C(M)$ is in dual form, the equivalence induced by Poincaré duality may be taken to be plus/minus the identity on four of the six modules. However, some redundancy in the proof of this, together with evidence on the level of the derived category of $\mathbb{Z}[G]$ on the one hand, and the homology of $\tilde{M}$ on the other, suggest that we may extend this to all six modules.

Received June 22, 2015; published on March 29, 2017.

2010 Mathematics Subject Classification: 57P10, 57M60, 55U15, 55N91, 55N45.

Key words and phrases: manifold, Poincaré duality, chain complex.

Article available at http://dx.doi.org/10.4310/HHA.2017.v19.n1.a9

Copyright (C) 2017, International Press. Permission to copy for private use granted. 
In this case $C(M)$ would satisfy a stronger duality and we say $M$ satisfies antiself-duality. Here the modules in $C(M)$ and $C(M)^{*}$ are again identified, with four of the five differentials the same, but now the remaining differential differs by a sign in the two cases. Finally we find an obstruction to anti-self-duality and show that not all 5-manifolds satisfy it, whilst providing a class of examples which do.

\section{Notation}

Fix a finite group $G$. All modules are right modules unless otherwise stated and assumed to be over $\mathbb{Z}[G]$, as are linear maps between modules. Bilinear forms on modules are assumed to be $G$ invariant. We abbreviate finitely generated to fg.

By algebraic complex we mean a sequence of modules and maps (referred to as differentials) where the composition of successive terms is 0 . The maps will be represented by solid arrows. Dashed arrows will denote identifications of the kernel/cokernel of the maps at either end with a module. Dashed arrows should not be regarded as part of the complex (for homotopies between chain maps etc.).

By a simple homotopy equivalence we mean interchanging an algebraic complex with one which differs from it in only two consecutive modules, where isomorphic free modules have been added to both modules, and the differential between them has been extended by the isomorphism. The preceding/following differentials are composed with the natural inclusion/projection respectively. If two algebraic complexes are related in this way, then we have a projection chain map and an inclusion chain map between them, which form a pair of mutually inverse chain homotopy equivalences.

Definition 1.1. For a finite algebraic complex $\mathcal{A}$, the $n$th stabilization of $\mathcal{A}$, denoted $\mathcal{A}^{n}$, is the algebraic complex obtained by adding a free rank $n$ module $F$ to the leftmost module in $\mathcal{A}$ (with maps written going from left to right). The differential from this module is extended to 0 on $F$.

We define the dual of a right $\mathbb{Z}[G]$ module $X$ to be the right $\mathbb{Z}[G]$ module $X^{*}$ obtained by taking the left $\mathbb{Z}[G]$ module $\operatorname{Hom}_{\mathbb{Z}}(X, \mathbb{Z})$ and defining the right action of $g \in G$ to be left multiplication by $g^{-1}$.

We extend this definition in the natural way to linear maps and algebraic complexes. Note that dualization preserves exactness at a module in an algebraic complex of torsion free (over $\mathbb{Z}$ ) fg $\mathbb{Z}[G]$ modules. We do not introduce signs when we dualize differentials. The price for this is a sign factor in the statement of Poincaré duality (see (8)). Define $H^{i}(\mathcal{A})=H_{i}\left(\mathcal{A}^{*}\right)$ for an algebraic complex $\mathcal{A}$.

The integers $\mathbb{Z}$ are a module over $\mathbb{Z}[G]$ with trivial $G$ action. A bilinear form $X \times X \rightarrow \mathbb{Z}$ will be referred to interchangeably as a map $X \rightarrow X^{*}$.

Let TOP $^{5}$ denote the category of closed connected oriented five dimensional topological manifolds with base point, equipped with an identification of their fundamental group with $G$. Let the morphisms in TOP $^{5}$ be the continuous maps which preserve the base point and induce the identity on $G$.

Given an object $M \in \mathrm{TOP}^{5}$ we may find a finite CW complex $M^{\prime}$ which is homotopy equivalent to it $\left[\mathbf{3}\right.$, Ann. 2]. Let $C_{*}\left(\tilde{M}^{\prime}\right)$ be the (cellular) chain complex of $\tilde{M}^{\prime}$ :

$$
C_{*}\left(\tilde{M}^{\prime}\right):=C_{5} \stackrel{\partial_{5}}{\longrightarrow} C_{4} \stackrel{\partial_{4}}{\longrightarrow} C_{3} \stackrel{\partial_{3}}{\longrightarrow} C_{2} \stackrel{\partial_{2}}{\longrightarrow} C_{1} \stackrel{\partial_{1}}{\longrightarrow} C_{0} .
$$

This is an algebraic complex of fg free $\mathbb{Z}[G]$ modules and $\mathbb{Z}[G]$-linear maps. It is exact 
at $C_{1}$ and $\operatorname{coker}\left(\partial_{1}\right) \cong \mathbb{Z}$ (as $M$ connected). We fix this isomorphism by fixing the homology class represented by a point to correspond to $1 \in \mathbb{Z}$.

Poincaré duality states that $C_{*}\left(\tilde{M}^{\prime}\right)$ is chain homotopy equivalent to its dual $C_{*}\left(\tilde{M}^{\prime}\right)^{*}$. Therefore it is exact at $C_{4}$ and $\operatorname{ker}\left(\partial_{5}\right) \cong \mathbb{Z}$. We fix this isomorphism by demanding the generator of $\operatorname{ker}\left(\partial_{5}\right)$ preferred by the orientation, maps to $1 \in \mathbb{Z}$.

The Euler characteristic (alternating sum of torsion free ranks over $\mathbb{Z}$ ) of $C_{*}\left(\tilde{M}^{\prime}\right)$ is minus that of $C_{*}\left(\tilde{M}^{\prime}\right)^{*}$ (as dualization preserves the torsion free $\mathbb{Z}$ rank of an $\mathrm{fg}$ $\mathbb{Z}[G]$ module). However, if they are chain homotopy equivalent they must also have equal Euler characteristic, which must then be 0 .

Definition 1.2. Let $\mathrm{ALG}^{5}$ denote the category of algebraic 5-complexes of fg free $\mathbb{Z}[G]$ modules:

$$
\mathbb{Z}-\rightarrow F_{5} \stackrel{\partial_{5}}{\longrightarrow} F_{4} \stackrel{\partial_{4}}{\longrightarrow} F_{3} \stackrel{\partial_{3}}{\longrightarrow} F_{2} \stackrel{\partial_{2}}{\longrightarrow} F_{1} \stackrel{\partial_{1}}{\longrightarrow} F_{0}-\rightarrow \mathbb{Z},
$$

satisfying exactness at $F_{4}$ and $F_{1}$, and with Euler characteristic 0 . Define the morphisms in this category to be homotopy classes of chain maps.

We may define a functor $C: \mathrm{TOP}^{5} \rightarrow \mathrm{ALG}^{5}$ by choosing a homotopy equivalence, $h_{M}: M \rightarrow M^{\prime}$ with $M^{\prime}$ a finite $\mathrm{CW}$ complex, for each $M \in \mathrm{TOP}^{5}$. In each case fix also $h_{M}^{\prime}$ a homotopy inverse of $h_{M}$. Then define $C(M)$ to be $C_{*}\left(\tilde{M}^{\prime}\right)$.

Given a morphism in $\mathrm{TOP}^{5}, f: M_{1} \rightarrow M_{2}$, we may select a cellular map $f^{\prime}: M_{1}^{\prime} \rightarrow$ $M_{2}^{\prime}$, which is homotopic to $\left(h_{M_{2}} \circ f \circ h_{M_{1}}^{\prime}\right)$. Define $C(f)$ to be the equivalence class of the chain map $f_{*}^{\prime}: C_{*}\left(\tilde{M}_{1}^{\prime}\right) \rightarrow C_{*}\left(\tilde{M}_{2}^{\prime}\right)$.

The isomorphism class of $C(M)$ in $\mathrm{ALG}^{5}$ is an invariant of $M$ as by construction different choices of $M^{\prime}$ must be homotopy equivalent to each other.

\section{Dual form}

Consider an algebraic 2-complex $\mathcal{B}$ of free fg $\mathbb{Z}[G]$ modules, where the cokernel of the last map is identified with $\mathbb{Z}$, we have exactness at the middle term and the dual of the kernel of the first map is denoted $J$ :

$$
\mathcal{B}:=\quad J^{*}-\stackrel{\iota}{\rightarrow} F_{2} \stackrel{d_{2}}{\longrightarrow} F_{1} \stackrel{d_{1}}{\longrightarrow} F_{0}-->\mathbb{Z} .
$$

Thus $J^{*} \in \Omega_{3}(\mathbb{Z})$ the third syzygy of $\mathbb{Z}[\mathbf{2}$, p6]. Equip $\mathcal{B}$ with a $G$ invariant bilinear form $\beta$ on $J$. We can associate to the pair $(\mathcal{B}, \beta)$ an algebraic 5 -complex:

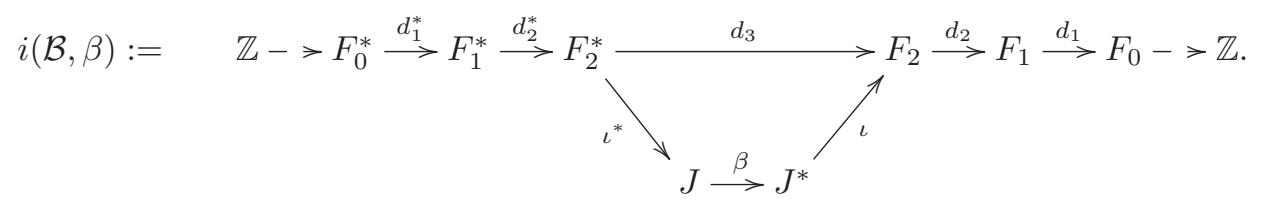

Let DUAL ${ }^{2}$ denote the category whose objects are pairs $(\mathcal{B}, \beta)$ as above.

We define the morphisms of $\mathrm{DUAL}^{2}$ to be homotopy equivalence classes of chain maps between the associated algebraic 5-complexes. If an object of $\mathrm{ALG}^{5}$ is of the form $i(\mathcal{B}, \beta)$, for some $(\mathcal{B}, \beta) \in \mathrm{DUAL}^{2}$ then we say it is in dual form.

Theorem 2.1. The functor $i$ is a natural equivalence of categories. 
By construction $i: \mathrm{DUAL}^{2} \rightarrow \mathrm{ALG}^{5}$ is a full faithful functor. It remains to show that every object in $\mathrm{ALG}^{5}$ is isomorphic to an object in the image of $i$. Our proof will follow stages analogous to the proof of [5, Theorem 1.1].

Consider an arbitrary element of $\mathrm{ALG}^{5}$ :

$$
\mathbb{Z}-->C_{5} \stackrel{\partial_{5}}{\longrightarrow} C_{4} \stackrel{\partial_{4}}{\longrightarrow} C_{3} \stackrel{\partial_{3}}{\longrightarrow} C_{2} \stackrel{\partial_{2}}{\longrightarrow} C_{1} \stackrel{\partial_{1}}{\longrightarrow} C_{0}-->\mathbb{Z} .
$$

We perform three pairs of simple homotopy equivalences. Firstly, the complex (1) is chain homotopy equivalent to

$$
C_{5} \oplus C_{0}^{*} \stackrel{\delta_{5}}{\longrightarrow} C_{4} \oplus C_{0}^{*} \stackrel{\partial_{4} \oplus 0}{\longrightarrow} C_{3} \stackrel{\partial_{3}}{\longrightarrow} C_{2} \stackrel{\partial_{2}}{\longrightarrow} C_{1} \oplus C_{5}^{*} \stackrel{\delta_{1}}{\longrightarrow} C_{0} \oplus C_{5}^{*},
$$

where

$$
\delta_{1}=\left(\begin{array}{cc}
\partial_{1} & 0 \\
0 & 1
\end{array}\right), \quad \delta_{5}=\left(\begin{array}{cc}
\partial_{5} & 0 \\
0 & 1
\end{array}\right) .
$$

Let $R_{0}=C_{0}, R_{5}=C_{5}$, and $R_{1}=C_{1} \oplus C_{5}^{*}, R_{4}=C_{4} \oplus C_{0}^{*}$. Rewrite (2):

$$
R_{5} \oplus R_{0}^{*} \stackrel{\delta_{5}}{\longrightarrow} R_{4} \stackrel{\partial_{4} \oplus 0}{\longrightarrow} C_{3} \stackrel{\partial_{3}}{\longrightarrow} C_{2} \stackrel{\partial_{2}}{\longrightarrow} R_{1} \stackrel{\delta_{1}}{\longrightarrow} R_{0} \oplus R_{5}^{*} .
$$

Again we perform a pair of simple homotopy equivalences. The complex (3) is chain homotopy equivalent to

$$
R_{5} \oplus R_{0}^{*} \stackrel{\delta_{5}}{\longrightarrow} R_{4} \oplus R_{1}^{*} \stackrel{\delta_{4}}{\longrightarrow} C_{3} \oplus R_{1}^{*} \stackrel{\partial_{3} \oplus 0}{\longrightarrow} C_{2} \oplus R_{4}^{*} \stackrel{\delta_{2}}{\longrightarrow} R_{1} \oplus R_{4}^{*} \stackrel{\delta_{1} \oplus 0}{\longrightarrow} R_{0} \oplus R_{5}^{*},
$$

where

$$
\delta_{2}=\left(\begin{array}{cc}
\partial_{2} & 0 \\
0 & 1
\end{array}\right), \quad \delta_{4}=\left(\begin{array}{cc}
\partial_{4} \oplus 0 & 0 \\
0 & 1
\end{array}\right) .
$$

Let $R_{2}=C_{2} \oplus R_{4}^{*}, R_{3}=C_{3} \oplus R_{1}^{*}$. Then (4) can be written:

$$
R_{5} \oplus R_{0}^{*} \stackrel{\delta_{5}}{\longrightarrow} R_{4} \oplus R_{1}^{*} \stackrel{\delta_{4}}{\longrightarrow} R_{3} \stackrel{\partial_{3} \oplus 0}{\longrightarrow} R_{2} \stackrel{\delta_{2}}{\longrightarrow} R_{1} \oplus R_{4}^{*} \stackrel{\delta_{1} \oplus 0}{\longrightarrow} R_{0} \oplus R_{5}^{*} .
$$

As all the modules in this complex are free and the Euler characteristic is 0, we can assume the existence of some isomorphism $\theta: R_{2}^{*} \rightarrow R_{3}^{*}$.

We perform a final simple homotopy equivalence to get:

$$
R_{5} \oplus R_{0}^{*} \stackrel{\delta_{5}}{\longrightarrow} R_{4} \oplus R_{1}^{*} \stackrel{\delta_{4}}{\longrightarrow} R_{3} \oplus R_{2}^{*} \stackrel{\delta_{3}}{\longrightarrow} R_{2} \oplus R_{3}^{*} \stackrel{\delta_{2} \oplus 0}{\longrightarrow} R_{1} \oplus R_{4}^{*} \stackrel{\delta_{1} \oplus 0}{\longrightarrow} R_{0} \oplus R_{5}^{*},
$$

where

$$
\delta_{3}=\left(\begin{array}{cc}
\partial_{3} \oplus 0 & 0 \\
0 & \theta
\end{array}\right)
$$

Our initial algebraic complex (1) is therefore chain homotopy equivalent to (5). We will show that (5) is chain isomorphic to an algebraic 5-complex in dual form. 
Lemma 2.2. There exist a pair of inverse chain isomorphisms $h, k$ :

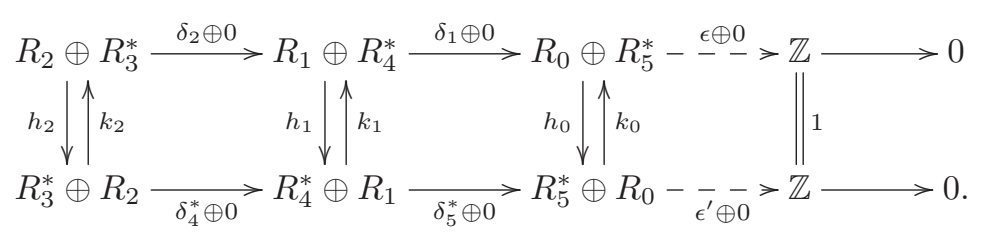

Proof. This is just a case of [5, Lemma 2.1].

Let $d_{3}=\delta_{3} k_{2}^{*}$ and write $(6)$ as

$$
S_{2} \stackrel{d_{2}}{\longrightarrow} S_{1} \stackrel{d_{1}}{\longrightarrow} S_{0} .
$$

Lemma 2.3. The complex (5) is chain isomorphic to

$$
S_{0}^{*} \stackrel{d_{1}^{*}}{\longrightarrow} S_{1}^{*} \stackrel{d_{2}^{*}}{\longrightarrow} S_{2}^{*} \stackrel{d_{3}}{\longrightarrow} S_{2} \stackrel{d_{2}}{\longrightarrow} S_{1} \stackrel{d_{1}}{\longrightarrow} S_{0} .
$$

Proof. We have the following chain isomorphism:

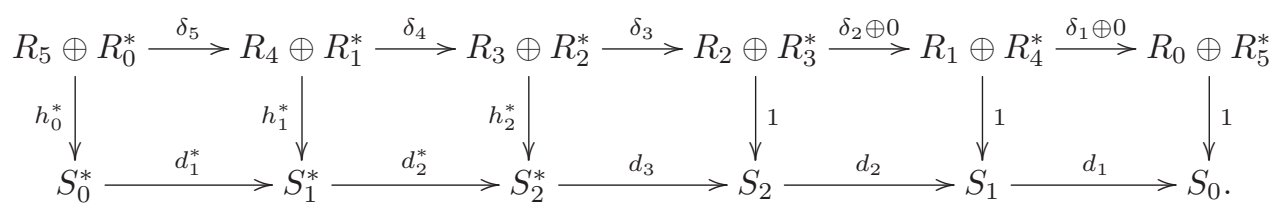

We may verify that the central square commutes: $d_{3} h_{2}^{*}=\delta_{3} k_{2}^{*} h_{2}^{*}=\delta_{3}$.

Clearly $d_{3}$ is induced by a $G$ invariant bilinear form on the dual of $\operatorname{ker}\left(d_{2}\right)$. This then completes the proof of Theorem 2.1.

Thus when using the functor $C$ to provide an invariant (up to isomorphism) of an element of $\mathrm{TOP}^{5}$, we may work in the category DUAL ${ }^{2}$. From now on we will suppress the functor $i$ and regard $\mathrm{DUAL}^{2}$ as a full subcategory of $\mathrm{ALG}^{5}$.

Note that if $(\mathcal{B}, \beta) \in$ DUAL $^{2}$, and $\mathcal{B}^{\prime}$ is related to $\mathcal{B}$ via a simple homotopy equivalence (recall definition from $\S 1$ ) then $(\mathcal{B}, \beta)$ is related to $\left(\mathcal{B}^{\prime}, \beta\right)$ by a pair of simple homotopy equivalences.

Recall Definition 1.1. Then $(\mathcal{B}, \beta)$ and $\left(\mathcal{B}^{n}, \beta\right)$ are related by a simple homotopy equivalence, (where in the latter $\beta$ is understood to extend to the standard inner product on the free module which we regard as a direct sum of copies of $\mathbb{Z}[G]$ ).

In some sense $\mathcal{B}$ is not important. That is we may fix an algebraic 2 -complex $\mathcal{B}$, so that up to isomorphism any element of $\mathrm{DUAL}^{2}$ has the form $\left(\mathcal{B}^{n}, \beta\right)$ for some integer $n$ and bilinear form $\beta$. To that end fix an algebraic 2-complex:

$$
\mathcal{B}:=\quad J^{*}-->F_{2} \stackrel{d_{2}}{\longrightarrow} F_{1} \stackrel{d_{1}}{\longrightarrow} F_{0}-->\mathbb{Z},
$$

with $F_{0}, F_{1}, F_{2}$ fg free and exactness at $F_{1}$. Let $J_{n}$ denote $J \oplus \mathbb{Z}[G]^{n}$.

Theorem 2.4. Given $(\mathcal{C}, \gamma) \in \mathrm{DUAL}^{2}$ there exists an integer $n$ and bilinear form $\beta$ on $J_{n}$ such that $(\mathcal{C}, \gamma)$ is chain homotopy equivalent to $\left(\mathcal{B}^{n}, \beta\right)$. 
Proof. By [5, Lemma 2.1] there exists an integer $n$ such that we may apply a pair of simple homotopy equivalences to $\mathcal{B}^{n}$ to get a complex:

$$
L_{2} \stackrel{D_{2}}{\longrightarrow} L_{1} \stackrel{D_{1}}{\longrightarrow} L_{0},
$$

and a pair of simple homotopy equivalences to some stabilization of $\mathcal{C}$ to get:

$$
S_{2} \stackrel{\partial_{2}}{\longrightarrow} S_{1} \stackrel{\partial_{1}}{\longrightarrow} S_{0},
$$

such that there is a chain isomorphism:

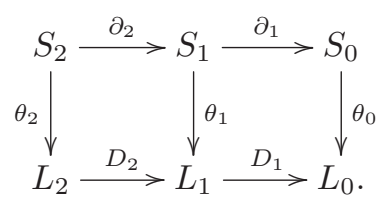

As noted above, $(\mathcal{C}, \gamma)$ is chain homotopy equivalent to

$$
S_{0}^{*} \stackrel{\partial_{1}^{*}}{\longrightarrow} S_{1}^{*} \stackrel{\partial_{2}^{*}}{\longrightarrow} S_{2}^{*} \stackrel{\partial_{3}}{\longrightarrow} S_{2} \stackrel{\partial_{2}}{\longrightarrow} S_{1} \stackrel{\partial_{1}}{\longrightarrow} S_{0},
$$

for some map $\partial_{3}$. Let $D_{3}=\theta_{2} \circ \partial_{3} \circ \theta_{2}^{*}$. We have a chain isomorphism:

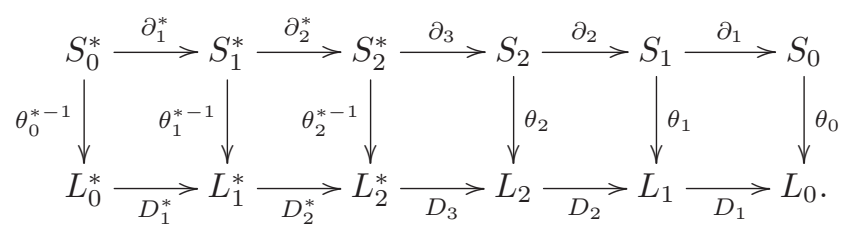

Finally note that $(7)$ is obtained from $\left(\mathcal{B}^{n}, \beta\right)$ for some $\beta$, by two pairs of simple homotopy equivalences. Hence $\left(\mathcal{B}^{n}, \beta\right)$ is chain homotopy equivalent to $(\mathcal{C}, \gamma)$.

So with $\mathcal{B}$ fixed, for any $M \in \mathrm{TOP}^{5}$ we have an integer $n$ and bilinear form $\beta$ on $J_{n}$, such that $C(M)$ is isomorphic to $\left(\mathcal{B}^{n}, \beta\right)$ in $\mathrm{ALG}^{5}$. In particular, the form $\beta$ encapsulates all the twisted (co)homology of $M$. A benefit of this is that one may attempt to classify objects in $\mathrm{ALG}^{5}$ and $\mathrm{TOP}^{5}$ without having to classify algebraic 2-complexes over $\mathbb{Z}[G]$ (an abstruse problem in its own right $[\mathbf{2}]$ ).

\section{Anti-self-duality}

Pick $\eta \in H_{5}(C(M))$ a generator. Poincaré Duality may be stated: There is a chain homotopy equivalence $\phi: C(M)^{*} \rightarrow C(M)$, such that for $\alpha \in H^{p}(C(M))$ we have:

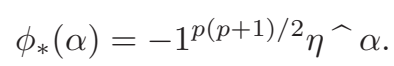

Given $\alpha \in H^{p}(C(M)), \gamma \in H^{5-p}(C(M))$ the graded symmetry of the cup product implies that $\gamma\left(\eta^{\wedge} \alpha\right)=\alpha\left(\eta^{\wedge} \gamma\right)$. Substituting (8) into this we get:

$$
\gamma\left(\phi_{*}(\alpha)\right)=-\alpha\left(\phi_{*}(\gamma)\right)
$$

Suppose, that we have a chain homotopy equivalence $f: C(M) \rightarrow \mathcal{A}$, for some $\mathcal{A} \in \mathrm{ALG}^{5}$. We have a chain homotopy equivalence $\phi^{\prime}=f \circ \phi \circ f^{*}: \mathcal{A}^{*} \rightarrow \mathcal{A}$. 
Definition 3.1. If a homotopy equivalence $\mathcal{A}^{*} \rightarrow \mathcal{A}$ is chain homotopy equivalent to one constructed in this way for some $f$, we say it is a duality equivalence.

Given $\alpha \in H^{p}(\mathcal{A}), \gamma \in H^{5-p}(\mathcal{A})$ we have $\gamma\left(\phi_{*}^{\prime}(\alpha)\right)=f^{*}(\gamma)\left(\phi_{*} f^{*}(\alpha)\right)$ which is antisymmetric in $\alpha, \gamma$ (by (9)), so we may conclude $\gamma\left(\phi_{*}^{\prime}(\alpha)\right)=-\alpha\left(\phi_{*}^{\prime}(\gamma)\right)$.

By Theorem 2.1 we may choose $\mathcal{A}$ to be of the form $(\mathcal{B}, \beta)$ for some

$$
\mathcal{B}:=\quad J^{*}-\stackrel{\iota}{-}>F_{2} \stackrel{d_{2}}{\longrightarrow} F_{1} \stackrel{d_{1}}{\longrightarrow} F_{0}-->\mathbb{Z},
$$

and bilinear form $\beta: J \times J \rightarrow \mathbb{Z}$. Let $d_{3}=\iota \beta \iota^{*}$.

The duality equivalence $\phi^{\prime}:(\mathcal{B}, \beta)^{*} \rightarrow(\mathcal{B}, \beta)$ may be written:

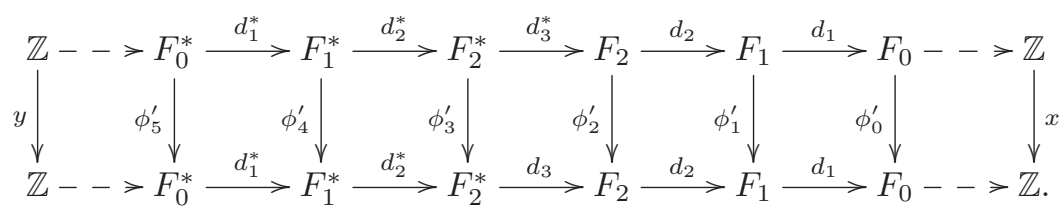

The natural pairing $F_{0}^{*} \times F_{0} \rightarrow \mathbb{Z}$ induces a pairing on the copies of $\mathbb{Z}$ on the left of this diagram, with those on the right. We set the generators $1 \in \mathbb{Z}$ on the right and left so that this pairing is given by multiplication. Let $\alpha \in H^{5}((\mathcal{B}, \beta)), \gamma \in H^{0}((\mathcal{B}, \beta))$ correspond to these generators.

We have $x=\gamma\left(\phi_{*}^{\prime}(\alpha)\right)=-\alpha\left(\phi_{*}^{\prime}(\gamma)\right)=-y$. Without loss of generality we may assume (by for example replacing $d_{3}$ with $-d_{3}$ ), that $x=1, y=-1$.

Theorem 3.2. For some maps $\theta_{1}, \theta_{2}$ we have a duality equivalence:

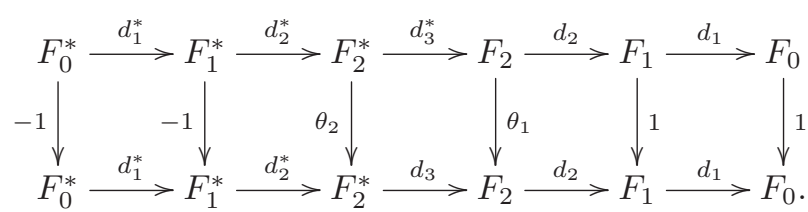

Proof. By the projectivity of the $F_{i}$ we may define maps $I_{0}, I_{1}, I_{3}, I_{4}$ :

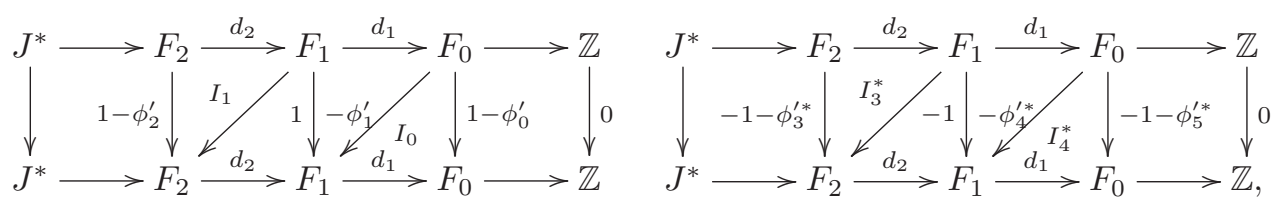

such that

$$
\begin{aligned}
d_{1} I_{0}=1-\phi_{0}^{\prime}, & I_{0} d_{1}+d_{2} I_{1}=1-\phi_{1}^{\prime}, \\
d_{1} I_{4}^{*}=-1-\phi_{5}^{*}, & I_{4}^{*} d_{1}+d_{2} I_{3}^{*}=-1-\phi_{4}^{\prime *} .
\end{aligned}
$$

Set

$$
\theta_{1}=\phi_{2}^{\prime}+I_{1} d_{2}, \quad \theta_{2}=\phi_{3}^{\prime}+d_{2}^{*} I_{3}, \quad I_{2}=0 .
$$

Then the $I_{i}, i=0,1,2,3,4$ form the required chain homotopy (10) to (11).

Theorem 2.1 tells us that we may always pick a representative $\mathcal{A}=(\mathcal{B}, \beta)$ of the homotopy type of $C(M)$ such that the modules of $\mathcal{A}$ and $\mathcal{A}^{*}$ may be identified, with 
four of the five differentials identical. Theorem 3.2 tells us that Poincaré duality induces a homotopy equivalence $\mathcal{A}^{*} \rightarrow \mathcal{A}$ which is plus/minus the identity on four of the six maps. The natural completion of these results would be that we could also take $\theta_{1}=1, \theta_{2}=-1$ (which would imply $d_{3}^{*}=-d_{3}$ ).

Definition 3.3. Say that $M \in \mathrm{TOP}^{5}$ is anti-self-dual if we can pick $\mathcal{A}$ as above with $\theta_{1}=1, \theta_{2}=-1$.

Indeed in (12) we arbitrarily set $I_{2}=0$, so it may seem that a more careful choice of $I_{2}$ could lead to $\theta_{1}=1, \theta_{2}=-1$. There are two further reasons to conjecture that all $M \in \mathrm{TOP}^{5}$ are anti-self dual. These arise in the two themes which we seek to marry in this paper: the derived category of the fundamental group of $M$ and the homology of the simply connected manifold $\tilde{M}$.

Let $\lambda_{1}: J \rightarrow J$ be the dual of the map induced by $\theta_{1}$ and let $\lambda_{2}: J \rightarrow J$ be the map induced by $\theta_{2}$. Commutativity in the central square of (11) implies that for all $u, v \in J: \beta\left(\lambda_{1} u, v\right)=\beta\left(\lambda_{2} v, u\right)$.

Thus if we had $\lambda_{1}=1_{J}, \lambda_{2}=-1_{J}$ then $\beta$ would be antisymmetric. What we do know from Theorem 3.2 is that this is true at level of the derived category of $G$ (in the sense of $[\mathbf{2}$, Chapter 4$]) ; \lambda_{1}, \lambda_{2}$ augment to $1,-1 \in \mathbb{Z} /|G|$ respectively.

On the other hand $\operatorname{Tor}\left(H^{3}(\tilde{M})\right)$ may be naturally identified with a subquotient of $J \otimes \mathbb{Q}$. With respect to this identification, the linking form on $\operatorname{Tor}\left(H^{3}(\tilde{M})\right)$ (taking values in $\mathbb{Q} / \mathbb{Z})$ is induced by $\beta\left(\lambda_{1-},-\right)[\mathbf{1}, \mathbf{7}]$. From $[\mathbf{1}$, Lemma $\mathrm{D}(\mathrm{ii})]$ we know that this is antisymmetric.

Despite all this, it transpires that not all manifolds are anti-self-dual. An obstruction arises from a combination of a condition on $G$ and a condition on $H_{*}(\tilde{M})$.

Theorem 3.4. Let $M \in \mathrm{TOP}^{5}$. If $G$ has even order and $H_{3}(\tilde{M})$ has even rank over $\mathbb{Z}$, then $M$ is not anti-self-dual.

Proof. Suppose $M$ is anti-self-dual. Then we have that $C(M)$ is chain homotopy equivalent to $(\mathcal{B}, \beta)$, with $\beta$ an antisymmetric form on a module $J$ with $J^{*} \in \Omega_{3}(\mathbb{Z})$.

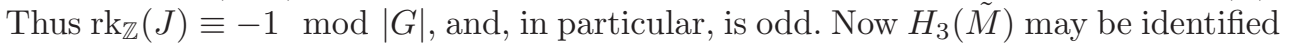
naturally with the kernel of $\beta$.

Thus we may split (over $\mathbb{Z}) J=H_{3}(\tilde{M}) \oplus J^{\prime}$ and extend $\beta$ to the tensor product $V=J^{\prime} \otimes \mathbb{Q}$. Then $V$ is an odd dimensional vector space over $\mathbb{Q}$, equipped with a non-degenerate anti-symmetric bilinear form. This is impossible.

As an example let $M$ be the Lens space $L(n ; 1,1)$. This has fundamental group $C_{n}=\left\langle t \mid t^{n}=1\right\rangle$. Let $\Sigma$ denote the sum of the $t^{i}$. We have $\mathcal{A}$ in dual form, with a chain homotopy equivalence $C(M) \rightarrow \mathcal{A}$ yielding the duality morphism $\phi$ :
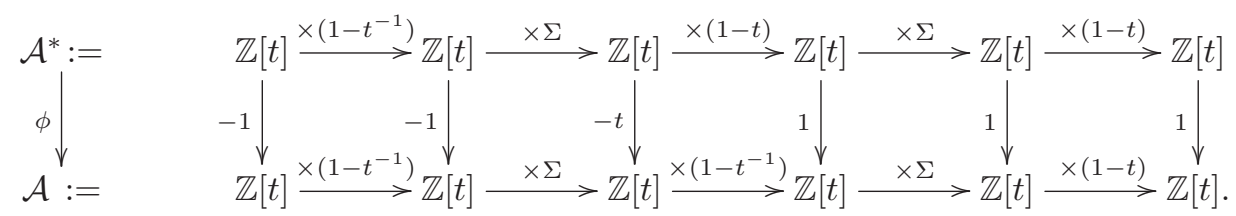

Note that dualizing multiplication by an element of $\mathbb{Z}[t]$ corresponds to involuting the element (e.g., substituting $t^{-1}$ for $t$ ). This results in the change in sign between the components of $\phi$ on the left and right sides, implied by Theorem 3.2. 
Note that the fourth component (from the right) of $\phi$ is $-t$, not -1 . If $n$ is even then this is unavoidable; $\tilde{M}=S^{5}$ so we know $H_{3}(\tilde{M})=0$ and Theorem 3.4 implies that $M$ is not anti-self-dual. Conversely:

Proposition 3.5. If $n=4 k+1$ then $M$ is anti-self-dual.

Proof. Let

$$
\alpha=t^{k+1}+t^{k}-t^{-k}-t^{-(k+1)}, \quad \beta=\sum_{r=-k+1}^{k} t^{r}-\sum_{r=k+2}^{3 k} t^{r} .
$$

Then $\alpha$ generates the same ideal as $t-1$, as $\alpha\left(t^{1+k}+t^{1-k}\right)=t^{2}-1$, which when multiplied by $1+t^{2}+t^{4}+\cdots+t^{4 k}$ returns $t-1$.

Now $\beta\left(1-t^{-1}\right)=\alpha$ and $\Sigma \beta=\Sigma$. Thus $\gamma \beta\left(1-t^{-1}\right)=\left(1-t^{-1}\right)$ for some $\gamma$, and $\gamma \beta=1+s \Sigma$ for some integer $s$. Then $(\gamma-s \Sigma) \beta=1$ and $\beta$ is a unit. Thus we have a chain homotopy equivalence $f: \mathcal{A} \rightarrow \mathcal{A}^{\prime}$ :
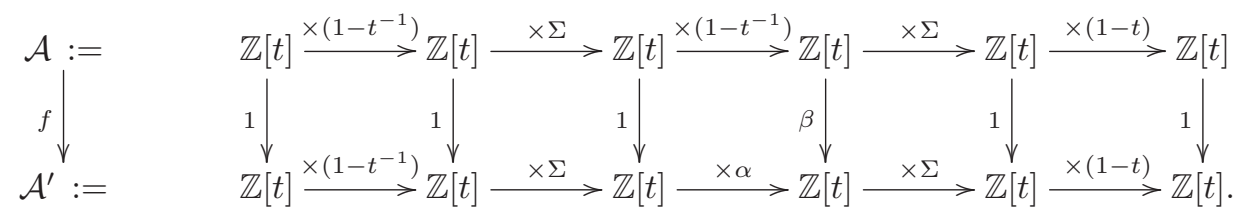

The chain homotopy equivalence $f \phi f^{*}$ is chain homotopic to the chain map whose first three components are 1 and whose last three components are -1 . The required homotopy has only one non-zero component; $I_{2}=x$, where $\alpha x=\beta-1$.

\section{References}

[1] D. Barden, Simply connected five-manifolds, The Annals of Mathematics 2nd Series 82 (3) (Nov., 1965), 365-385.

[2] F.E.A. Johnson, Stable modules and the D(2) problem, LMS 301, Cambridge University Press, Cambridge, 2003.

[3] R Kirby and L Siebenmann, Foundational essays on topological manifolds, smoothings and triangulations, Annals of Mathematics Studies 88, Princeton University Press, Princeton, NJ, 1977.

[4] J. Klein, Poincaré duality spaces, Surveys on surgery theory, Vol. 1, Annals of Mathematics Studies 145, 2000, pp. 135-165.

[5] W.H. Mannan, Homotopy types of truncated projective resolutions, Homology, Homotopy and Applications 9 (2) (2007), 445-449.

[6] C.T.C. Wall, Surgery on compact manifolds, AMS Mathematical Surveys and Monographs 69, 2nd edition, Providence, RI, 1999.

[7] C.T.C. Wall, Quadratic forms on finite groups and related topics, Topology 2 (1963), 281-298.

W.H. Mannan wajid@mannan.info

Department of Mathematics, City University London, Northampton Square, London, EC1V 0HB 